\title{
Effect of Methanol in the Wash-Solvent Bottle and the OnGuard Cartridges on the Determination of Different Anions Using Ion Chromatography
}

\author{
Adnan Manassra, Mustafa Khamis, Fida Obeidi, Majdi Dakiky, Fuad Al-Rimawi* \\ Faculty of Science and Technology, Al-Quds University, East Jerusalem, Palestine \\ Email: "falrimawi@science.alquds.edu
}

Received November 27, 2011; revised December 30, 2011; accepted January 9, 2012

\begin{abstract}
The effect of using methanol in the flush-solvent bottle of the auto-sampler of Ion Chromatograph on the determination of sulfate ions was studied using AS11-HC $2 \mathrm{~mm}$ analytical column. The use of 50\% methanol in the flush solvent bottle results in overestimation of sulfate concentration by $18 \%$. However, the use of $10 \%$ methanol instead of $50 \%$ did not show such error in the determination of sulfate using the same column. Furthermore, the effect of using OnGuard-RP and OnGuard-P cartridges for sample pretreatment on the determination of different anions was also studies using AS14-HC $2 \mathrm{~mm}$ analytical column. The results indicate that using OnGuard-RP cartridge caused the concentration of fluoride, bromide, nitrate, phosphate and sulfate ions to be underestimated, whereas the concentration of chloride ions to be overestimated. The error in the determination of fluoride, bromide, nitrate, phosphate sulfate, and chloride was found to be $40.0 \%, 26.0 \%, 22.8 \%, 26.8 \%, 18.0 \%$, and $25.0 \%$ of the certified standard concentration, respectively. It was also found that the use of OnGuard-P cartridge resulted in underestimation of fluoride, chloride, bromide, nitrate, phosphate, and sulfate by $60.0 \%, 40.0 \%, 82.0 \%, 87.2 \%, 45.2 \%$, and $39.0 \%$ of the certified standard concentration, respectively. These findings point out to careful corrections that should be considered in any application of ion chromatography utilizing such sample pretreatment.
\end{abstract}

Keywords: Ion Chromatography; Interference; Anions; Methanol; OnGuard Cartridges

\section{Introduction}

The number of species that may be determined by using ion chromatography (IC) is continuing to grow, as does the number of areas of science and technology. Nowadays, IC is being widely used for the analysis of ionic species in pharmaceuticals, detergents, fertilizers, pesticides, soil, plants, drinking water, wastewater etc. [1-11].

In our laboratories at Al-Quds University, a Dionex ion chromatograph, DX-500 is used for the determination of anions and cations in water, wastewater, soil and plant samples. The system is provided with improved eluent delivery system of the GP (50) gradient pump, a developed ED (40) electrochemical detector to self-regenerating suppressor and an AS3500 autosampler that made the system a state of art ion chromatograph with superior analytical performance. The AS3500 autosampler is provided with a flush-solvent bottle containing a solvent to remove air bubbles that are produced in the injection syringe during the introduction of the sample to the analytical column which affects the sample injection volume leading to in-

\footnotetext{
"Corresponding author.
}

accuracy in measured concentrations. 50\% methanol (by volume) has been the solvent of choice in some laboratories [2].

OnGuard-P and OnGuard-RP cartridges are used to remove matrices that may interfere with ion chromatography applications [2]. These matrices shorten the lifetime of the analytical columns and/or lead to inaccuracy in ion determination. OnGuard-P is used for the removal of phenolic fractions of humic acids, azo-containing compounds, aromatic carboxylic acids and aromatic aldehydes from samples prior to analysis on the IC [2]. OnGuard-RP is used for the removal of aromatic dyes, aromatic carboxylic acids, hydrocarbons and surfactants from samples [2].

The objective of this paper is to study the effect of using methanol in the flush-solvent bottle of the autosampler of Ion Chromatograph instrument, and the effect of using two types of column cartridges which are used in Ion Chromatography (OnGuard-RP and OnGuard-P) on the determination of different anions (fluoride, chloride, bromide, nitrate, phosphate, and sulfate) by using ion chromatography. 


\section{Experimental}

\subsection{Chemicals and Materials}

All chemicals were of analytical grades. Sodium hydroxide (50\% solution, low carbonate), sodium bicarbonate, and sodium carbonate were from J.J Baker. Methanol and ethanol were of chromatographic grade and obtained from Fortrum. 18.2 M $\Omega$ water was used in all sample preparation. OnGuard-P and OnGuard-RP cartridges were obtained from Dionex. Certified anions standards were obtained also from Dionex.

\subsection{Instrumentation}

Dionex DX 500 ion chromatograph with GP 50 gradient pump and ED 40 electrochemical detector with conductivity mode was used for the anion analysis. The instrument was configured with autosampler AS3500. AS11-HC 2 $\mathrm{mm}$ and AS14-HC $2 \mathrm{~mm}$ were used as analytical columns. Deionized 18.2 M $\Omega$ water were generated from Milli-Q system (Millipore).

\subsection{Method}

Samples were introduced into the autosampler vials after passing through $0.45 \mu \mathrm{m}$ filter and/or the OnGuard cartridges prior to analysis. The instrument scheduled with the appropriate method and data were processed and controlled by PEAKNET 5.1 supplied by Dionex. The flush solvent bottle of the autosampler was filled either with water, $10 \%$ methanol (by volume), or $50 \%$ methanol (by volume).

Methanol interference experiments were conducted using a DX-500 ion chromatograph using an AS11-HC 2 $\mathrm{mm}$ analytical column. The analyte anions were passed through AG11-HC $2 \mathrm{~mm}$ guard column before being introduced to the AS11-HC $2 \mathrm{~mm}$ analytical column. The eluates were then sent through a self-generating suppressor (ASRS-II) before being detected by the conductivity cell. The ionic eluent used in these experiments was $30 \mathrm{mM}$ sodium hydroxide with a flow rate of 0.38 $\mathrm{ml} / \mathrm{min}$. On the other hand; the OnGuard interference was studied under the same conditions as used above except the AG14-HC $2 \mathrm{~mm}$ and AS14-HC $2 \mathrm{~mm}$ are used as the guard and analytical columns, respectively. The ionic eluent in this case was a mixture of sodium carbonate $(3.5 \mathrm{mM})$ and sodium bicarbonate $(1.0 \mathrm{mM})$ with a flow rate of $0.75 \mathrm{ml} / \mathrm{min}$.

\section{Results and Discussion}

\subsection{Methanol Interference}

The injection of the sample in IC using a flush-solvent bottle for the autosampler that contains $100 \%$ water results in air bubbles in the injection valve and hence affects the sample injection volume leading to inaccuracy in the determination of ions. To eliminate this problem, water was replaced by $50 \%$ methanol, which has been used for many years in a number of laboratories. However, it was found in the current study that methanol interferes with the determination of anions especially with sulfate on AS11-HC $2 \mathrm{~mm}$ and interferes with fluoride on AS14-HC $2 \mathrm{~mm}$ analytical columns. Figure 1 displays the chromatogrms of a Dionex-standard containing fluoride, chloride, sulfate, nitrate and phosphate analyzed on the AS11-HC $2 \mathrm{~mm}$ analytical column, first using deionized water in the flush-solvent bottle (Chromatogram A) and then using 50\% methanol (by volume) in the flushsolvent bottle (Chromatogram B). Inspection of

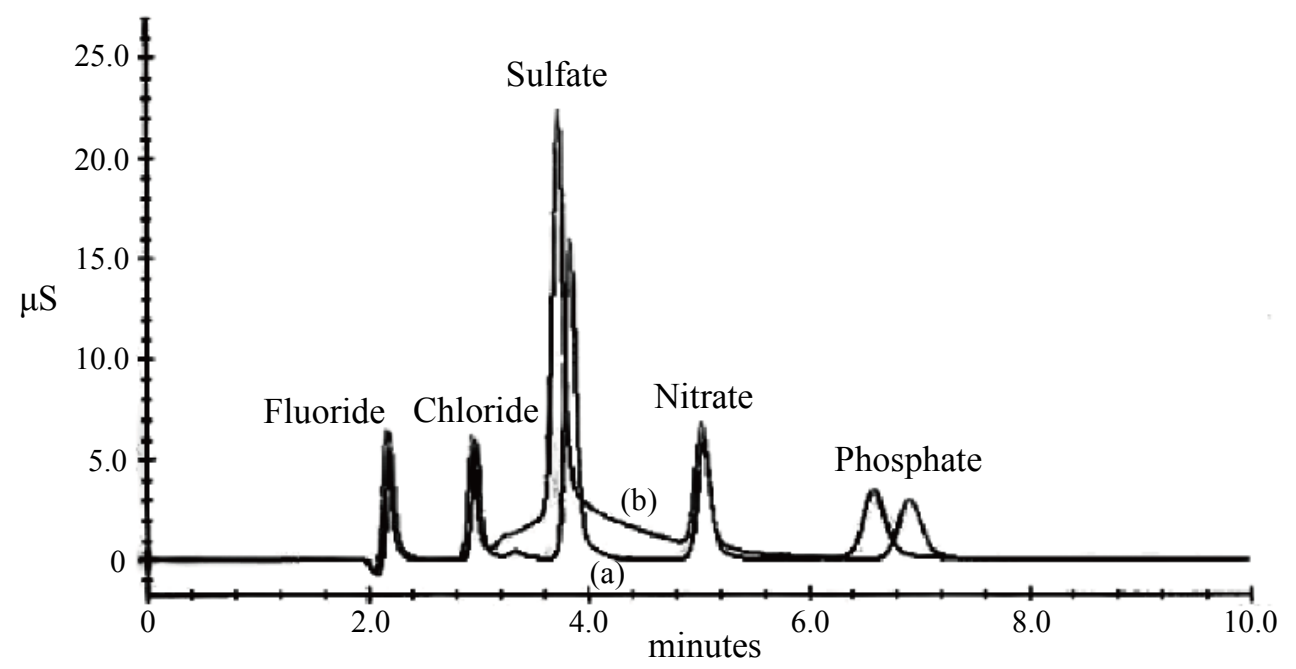

Figure 1. Chromatogram of fluoride, chloride, sulfate, nitrate, and phosphate on the AS11-HC 2 mm column using Ion Chromatograph DX500 configured with the AS3500 autosampler. (a) and (b) are chromatograms when $10 \%$ and $50 \%$ methanol (by volume) were used in the flush-solvent bottle of the injection system of the autosampler, respectively. 
these chromatograms reveals that the baseline near sulfate peak is ill-defined (not stable) when 50\% methanol is used resulting in overestimation of the sulfate concentration (Chromatogram B). In the process of identifying the source of the interference, and quantifying the overestimation in sulfate concentration, two sets of experiments were performed. First, different solutions of methanol (with different volume fractions ranging from $1 \%$ and $10 \%$ ) were analyzed on the Dionex IC using the same chromatographic conditions used for analysis of the ions but using $100 \%$ of deionized water $(18.2 \mathrm{M} \Omega)$ in the flush-solvent bottle. Results which are shown in Figure 2(a) indicates that methanol solutions ( $1 \%$ to $10 \%$ by volume) injected onto the IC have a peak with a retention time of 3.9 minutes which interferes with sulfate ion peak with retention time of 3.4 minutes under the same experimental conditions mentioned above. Results have also showed direct relationship between conductivity and volume fraction of methanol in the flush-solvent bottle (Figure 2(b)). A linear correlation was obtained until methanol concentration reaches $10 \%$ (by volume), Figure 2(b). These results explain the broadening of sulfate peak resulting from interference with methanol peak that is produced when traces of methanol in the flush-solvent bottle are injected along with the samples.

The second set of experiments which are performed to quantify the overestimation in sulfate concentration is analysis of deionized water samples (18.2 M $\Omega$ ) using $0 \%$ methanol (Figure 3(a)), 10\% methanol (Figure 3(b)), and 50\% methanol (Figure 3(c)) in the flush-solvent bottle. Inspection of Figure 3 reveals that the overestimation in sulfate concentration when $50 \%$ methanol (by volume) used is about $1.8 \mathrm{ppm}$ which is about $18 \%$ of the 10-ppm sulfate standard used. Figure 3(b) indicates low interference when $10 \%$ methanol is used; where the overestimation in sulfate concentration is only $0.1 \mathrm{ppm}$. In addition, no air bubbles were noticed in the injector syringe when using $10 \%$ methanol which minimizes the error resulting from inaccurate injection volume conesquential to using just water in the flush-solvent bottle.

\subsection{OnGuard Cartridges Interference}

To evaluate the interference resulting from OnGuard-RP and OnGuard-P cartridges, a Dionex standard containing fluoride, chloride, bromide, nitrate, phosphate and sulfate was analyzed on an AS14-HC $2 \mathrm{~mm}$ analytical column with and without using OnGuard cartridges prior to analysis (Figure 4). Figure 4(a) demonstrates the chromatogram of the Dionex-standard when OnGuard-RP cartridge was used, while Figure 4(b) illustrates the chromatogram of the same standard without treatment prior to analysis, and Figure 4(c) represent the chromatogram using OnGuard-P cartridge. When OnGuard-RP cartridge was used, the underestimation in fluoride, bromide, nitrate, phosphate, and sulfate concentrations, as compared to the values without pretreatment, were found to be $0.45,1.85$, $1.93,1.83$, and $4.1 \mathrm{ppm}$, respectively. This is equal to $40.0 \%, 26.0 \%, 22.8 \%, 26.8 \%$, and $18.0 \%$ of a standard containing $0.75,2.5,2.5,2.5$, and $5.0 \mathrm{ppm}$ of fluoride, bromide, nitrate, phosphate, and sulfate, respectively. On the other hand, it was found that chloride was overestimated by $0.25 \mathrm{ppm}$ in a standard containing $1 \mathrm{ppm}$ of chloride. When OnGuard-P cartridge was used, the underestimation in fluoride, chloride, bromide, nitrate, phosphate, and sulfate concentration was found to be more pronounced, as it is seen in Figure 4(c). The underestimation reached values of $0.3,0.6,0.45,0.32,1.37$, and 3.05 of a standard containing $0.75,1.0,2.5,2.5,2.5$, and $5.0 \mathrm{ppm}$ of fluoride, chloride, bromide, nitrate, phosphate, and sulfate, respectively which is equal to $60.0 \%, 40.0 \%$, $82.0 \%, 87.2 \%, 45.2 \%$, and $39.0 \%$. It is worth mentioning here that these experiments were conducted with $10 \%$ methanol in the flush-solvent bottle.

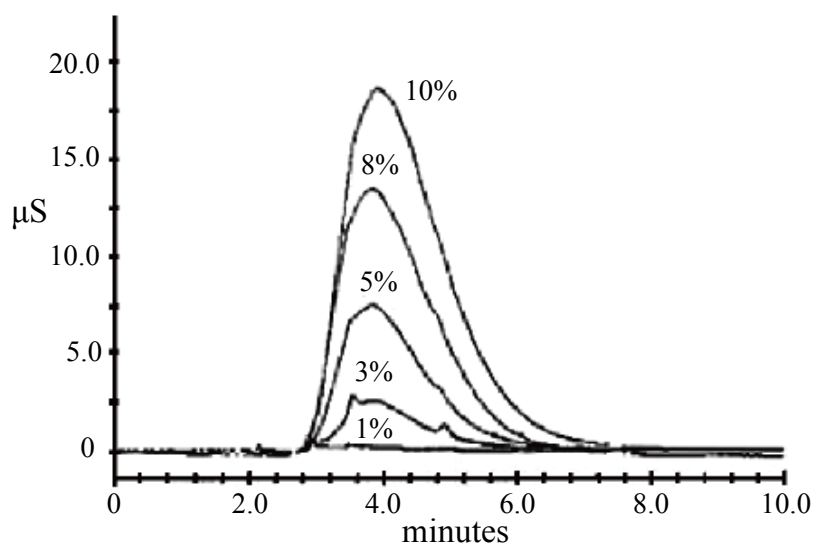

(a)

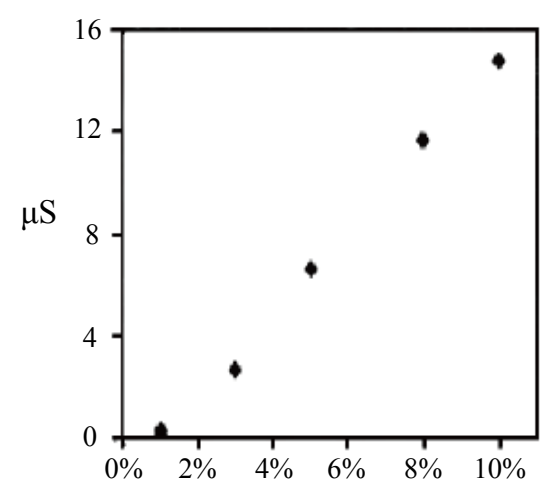

(b)

Figure 2. Chromatograms (a) and calibration curve (b) of different methanol solutions $(1 \%, 3 \%, 5 \%, 8 \%, 10 \%$, V/V) on the AS11-HC 2 mm analytical column configured with Guard column AG11-HC 2 mm. 


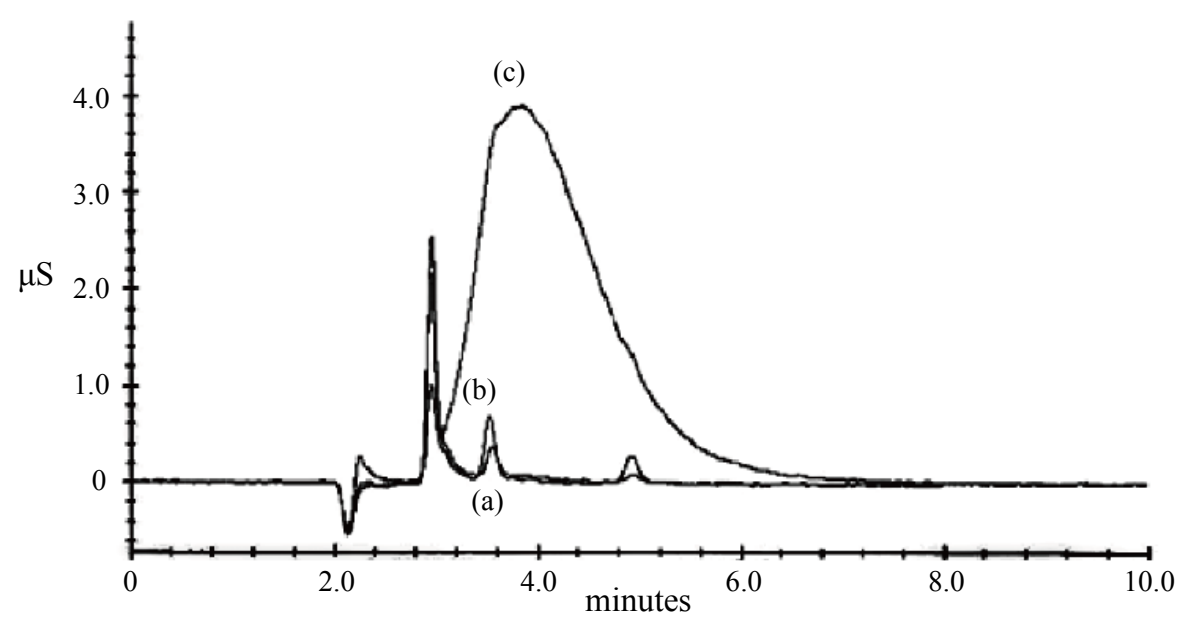

Figure 3. Chromatograms of water sample analyzed on AS11-HC 2 mm analytical column using Ion Chromatograph DX500 using $100 \%$ of deionized water in the flush-solvent bottle (chromatogram (a)), using $\mathbf{1 0 \%}$ methanol in the flush-solvent bottle (chromatogram (b)), and using $50 \%$ methanol in the flush-solvent bottle (chromatogram (c)).

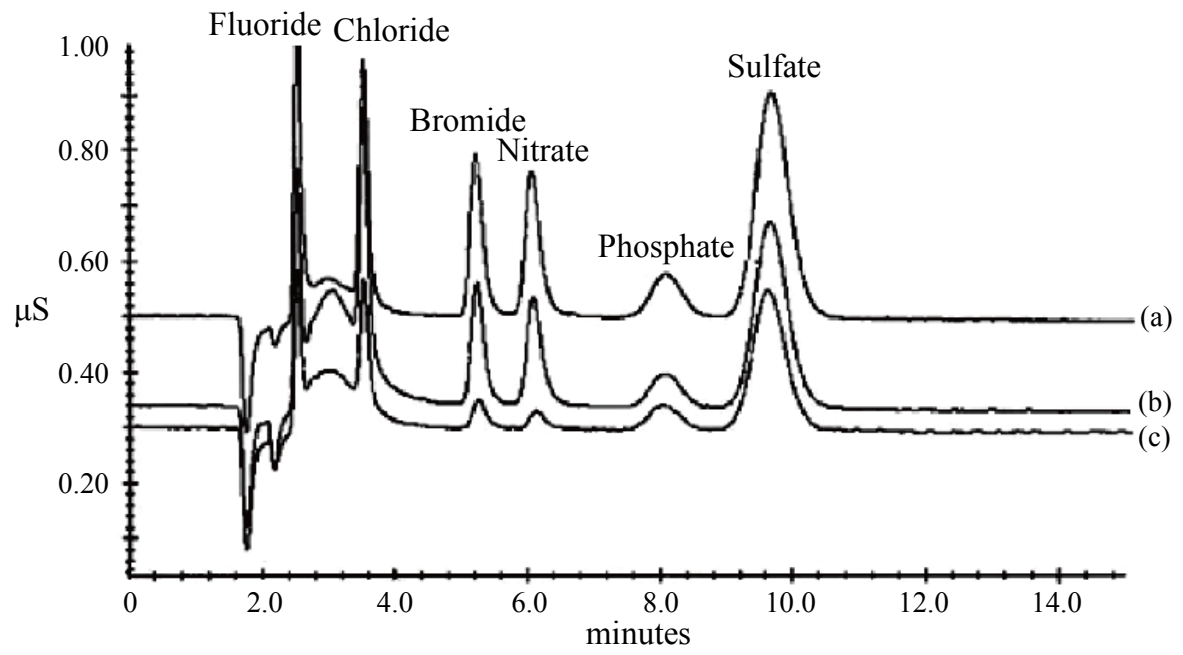

Figure 4. Chromatograms of Dionex standards on AS14-HC 2 mm column. (a) Sample was passed through the OnGuard-RP prior to analysis; (b) Without using OnGuard cartridges; (c) Using the OnGuard-P.

\section{Conclusion}

Water which is used in the flush-solvent bottle in the injecttion valve of the AS3500 autosampler of Ion Chromatograph instrument was replaced with methanol/water mixture to eliminate air bubbles in the injection syringe that may result in false injection volume which in turn lead to inaccurate results. $50 \%$ methanol in the flush-solvent bottle which is used in some laboratories interferes with anion determination by IC on both AS11-HC $2 \mathrm{~mm}$ and AS14HC $2 \mathrm{~mm}$ analytical columns and results in overestimation of sulfate ion concentration. Replacing 50\% methanol with $10 \%$ methanol decreases drastically the overestimation in sulfate determination and prevents the air bubbles in the injection valve. OnGuard cartridges (OnGuard-RP and OnGuard-P) used during sample pretreatment result in errors in anions determination. The use of OnGuard-RP cartridge leads to underestimation of fluoride, bromide, nitrate, phosphate, and sulfate, and overestimation of chloride. The use of OnGuard-P cartridge results also in the underestimation of fluoride, chloride, bromide, nitrate, phosphate, and sulfate but to a larger extent compared to the OnGuard-RP cartridge. The above results suggest that the final results of the IC determination using these techniques should be assessed carefully before reporting.

\section{REFERENCES}

[1] D. A. Skoog, F. J. Holler and S. R. Crouch, "Principles of Instrumental Analysis," Brooks/Cole, Belmont, 2007.

[2] P. R. Haddad and P. E. Jackson, "Ion Chromatography: Principles and Applications," Elsevier, New York, 1990.

[3] B. López-Ruiz, "Advances in the Determination of Inorganic Anions by Ion Chromatography," Journal of Chro- 
matography A, Vol. 881, No. 1-2, 2000, pp. 607-627. doi:10.1016/S0021-9673(00)00244-2

[4] C. A. Lucy, "Recent Advances in Ion Chromatography: A Perspective," Journal of Chromatography A, Vol. 739, No. 1-2, 1996, pp. 3-13. doi:10.1016/0021-9673(96)00034-9

[5] J. H. Lee, J. S. Kim, B. H. Min, S. T. Kim and J. H. Kim, "Determination of Anions in Certified Reference Materials by Ion Chromatography," Journal of Chromatography A, Vol. 813, No. 1, 1998, pp. 85-90. doi:10.1016/S0021-9673(98)00267-2

[6] P. N. Nesterenko, "Simultaneous Separation and Detection of Anions and Cations in Ion Chromatography," TrAC Trends in Analytical Chemistry, Vol. 20, No. 6-7, 2001, pp. 311-319. doi:10.1016/S0165-9936(01)00072-3

[7] R. García-Fernández, J. I. García-Alonso and A. SanzMedel, "Simultaneous Determination of Inorganic Anions, Calcium and Magnesium by Suppressed Ion Chromatography," Journal of Chromatography A, Vol. 1033, No. 1, 2004, pp. 127-133.

$$
\text { doi:10.1016/j.chroma.2004.01.024 }
$$

[8] J. S. Fritz, "Factors Affecting Selectivity in Ion Chromatography," Journal of Chromatography A, Vol. 1085, No. 1, 2005, pp. 8-17. doi:10.1016/j.chroma.2004.12.087

[9] P. R. Haddad, P. E. Jackson and M. J. Shaw, "Developments in Suppressor Technology for Inorganic Ion Analysis by Ion Chromatography Using Conductivity Detection," Journal of Chromatography A, Vol. 1000, No. 1-2, 2003, pp. 1903-2003. doi:10.1016/S0021-9673(02)01999-4

[10] W. Hu, H. Tao, M. Tominaga, A. Miyazaki and H. Haraguchi, "A New Approach for the Simultaneous Determination of Inorganic Cations and Anions Using Ion Chromatography," Analytica Chimica Acta, Vol. 299, No. 2, 1994, pp. 249-256. doi:10.1016/0003-2670(94)00347-5

[11] Y. Ding and S. Mou, "Effects of Common Metal Ions on the Determination of Anions by Suppressed Ion Chromatography," Journal of Chromatography A, Vol. 956, No. 1-2, 2002, pp. 65-70. doi:10.1016/S0021-9673(01)01545-X 